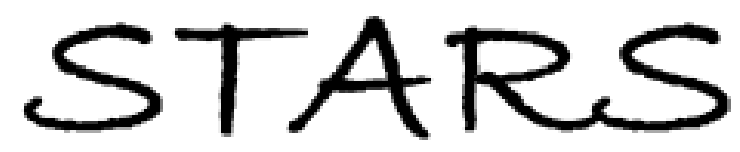

University of Central Florida

STARS

$1-1-2009$

\title{
Excitation of propagating surface plasmons by a periodic nanoparticle array: Trade-off between particle-induced near-field excitation and damping
}

Amitabh Ghoshal

University of Central Florida

Pieter G. Kik

University of Central Florida

Find similar works at: https://stars.library.ucf.edu/facultybib2000

University of Central Florida Libraries http://library.ucf.edu

This Article is brought to you for free and open access by the Faculty Bibliography at STARS. It has been accepted for inclusion in Faculty Bibliography 2000s by an authorized administrator of STARS. For more information, please contact STARS@ucf.edu.

\section{Recommended Citation}

Ghoshal, Amitabh and Kik, Pieter G., "Excitation of propagating surface plasmons by a periodic nanoparticle array: Trade-off between particle-induced near-field excitation and damping" (2009). Faculty Bibliography 2000s. 1570.

https://stars.library.ucf.edu/facultybib2000/1570

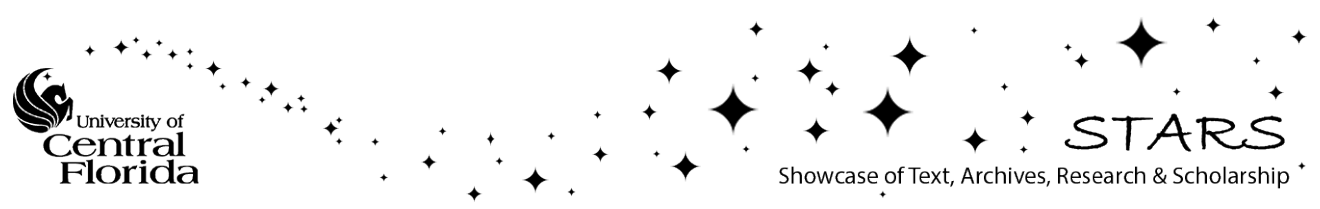




\section{Excitation of propagating surface plasmons by a periodic nanoparticle array: Trade- off between particle-induced near-field excitation and damping}

Cite as: Appl. Phys. Lett. 94, 251102 (2009); https://doi.org/10.1063/1.3156862

Submitted: 10 December 2008 . Accepted: 28 May 2009. Published Online: 22 June 2009

Amitabh Choshal, and Pieter G. Kik

\section{ARTICLES YOU MAY BE INTERESTED IN}

Theory and simulation of surface plasmon excitation using resonant metal nanoparticle arrays

Journal of Applied Physics 103, 113111 (2008); https://doi.org/10.1063/1.2936971

Dielectric stripes on gold as surface plasmon waveguides

Applied Physics Letters 88, 094104 (2006); https://doi.org/10.1063/1.2180448

Plasmonics: Localization and guiding of electromagnetic energy in metal/dielectric structures

Journal of Applied Physics 98, 011101 (2005); https://doi.org/10.1063/1.1951057

\section{Applied Physics Letters}

Mid-IR and THz frequency combs special collection 


\title{
Excitation of propagating surface plasmons by a periodic nanoparticle array: Trade-off between particle-induced near-field excitation and damping
}

\author{
Amitabh Ghoshal $^{\mathrm{a})}$ and Pieter G. Kik ${ }^{\mathrm{b})}$ \\ CREOL, The College of Optics and Photonics, University of Central Florida, 4000 Central Florida Blvd., \\ Orlando, Florida 32816, USA
}

(Received 10 December 2008; accepted 28 May 2009; published online 22 June 2009)

\begin{abstract}
The excitation of propagating surface plasmons (SPs) on a silver-silica interface by an array of ellipsoidal silver nanoparticles is investigated using numerical simulations as a function of particle volume for three different nanoparticle aspect ratios with representative resonance frequencies. We find that while the SP amplitude depends sensitively on particle volume for each selected aspect ratio, the maximum SP amplitude obtained for the different particle shapes is remarkably similar. These observations are explained in terms of particle-mediated SP excitation, counteracted by a size dependent particle-induced damping. An analytical model is presented that quantitatively describes the observed trends in SP damping. (C) 2009 American Institute of Physics.
\end{abstract}

[DOI: $10.1063 / 1.3156862$ ]

Surface plasmons (SPs) are electron charge density waves that can exist at a metal surface or at the interface between a metal and a dielectric. ${ }^{1}$ SPs can be strongly confined, making SPs on metal films possible candidates for nanoscale optical waveguides. ${ }^{2-6}$ Typical methods for exciting propagating SPs are (1) prism or grating coupling ${ }^{1}$ and (2) near-field excitation using isolated structures on or near a metal surface. ${ }^{7-10}$ We have previously both theoretically and experimentally studied SP excitation on a metal film using a nanoparticle (NP) enhanced grating coupler, and demonstrated strong coupling between the NP localized plasmon resonance and a grating resonance associated with the periodic particle arrangement. ${ }^{11,12}$ In the present work we numerically investigate the effect of changing the NP size on the excitation of SPs on the silver film. We demonstrate that the optimum particle volume for SP excitation depends sensitively on the particle resonance frequency.

The structure under investigation consists of a periodic array of ellipsoidal silver NPs placed near a silver film, as sketched in Fig. 1(a). This arrangement enables the constructive excitation of propagating SPs by NPs placed approximately one plasmon wavelength apart, while utilizing the NP plasmon resonance to modify the excitation efficiency. The system was studied using the Finite Integration Technique as implemented in CST MICROWAVE STUDIO. ${ }^{13} \mathrm{~A}$ detailed description of the simulation setup is available in Ref. 11. The silver film is $200 \mathrm{~nm}$ thick and extended infinitely in the $x-y$ plane, embedded in $\mathrm{SiO}_{2}$. An infinite array of ellipsoidal silver NPs of semiaxes $a \times b \times c$ along the $x, y$, and $z$-directions, respectively, is located in the $\mathrm{SiO}_{2}$ with the center of the NPs placed $80 \mathrm{~nm}$ above the metal surface. The NP aspect ratio (AR) is defined as $a / b$, and for all NPs the crosssection is circular, i.e., $c=b$. Due to the dipolar nature of the NP mode, maintaining a fixed particle-center height allows us to separately investigate the dependence of particle volume without the complicating influence of a varying dipoleto-surface distance. The system is excited using a pulsed $x$-polarized oscillating electric field $\left(E_{\text {in }}\right)$, with the pulse

\footnotetext{
a) Electronic mail: aghoshal@creol.ucf.edu.

${ }^{b)}$ Also at: Department of Physics, University of Central Florida.
}

propagating along the $z$-direction with a center frequency of $2.73 \times 10^{15} \mathrm{rad} / \mathrm{s}$ and a duration of $9.5 \mathrm{fs}$ [see inset, Fig. 1(a)]. The interparticle spacing in the $x$-direction $L_{x}$ $=440 \mathrm{~nm}$ coincides with the $\mathrm{Ag}-\mathrm{SiO}_{2} \mathrm{SP}$ wavelength at the center frequency. Fits to literature dielectric functions were used in the simulations, as described in detail in Ref. 11. A field probe monitors the normal field component $E_{z}$ just above the metal surface at position $\left(L_{x} / 4,0,1 \mathrm{~nm}\right)$ coinciding with the location of maximum SP amplitude. ${ }^{11} \mathrm{~A}$ relatively small interparticle spacing in the $y$-direction, $L_{y}$ $=100 \mathrm{~nm}$, was used to create a high particle density along the $y$-direction compared to that in the $x$-direction while keeping interparticle interactions low. This results in structures macroscopically similar to $y$-oriented line gratings.

Figure 1(a) shows the Fourier transform of the timedomain signal at the probe location, normalized to the Fourier transform of the incident pulse, for a $\mathrm{NP}$ with $\mathrm{AR}=3.5$ and volume $V_{\mathrm{NP}}=9.8 \times 10^{3} \mathrm{~nm}^{3}$. Two resonances are observed-one related to propagating SPs constructively excited due to the periodicity of the array (sharp resonance at

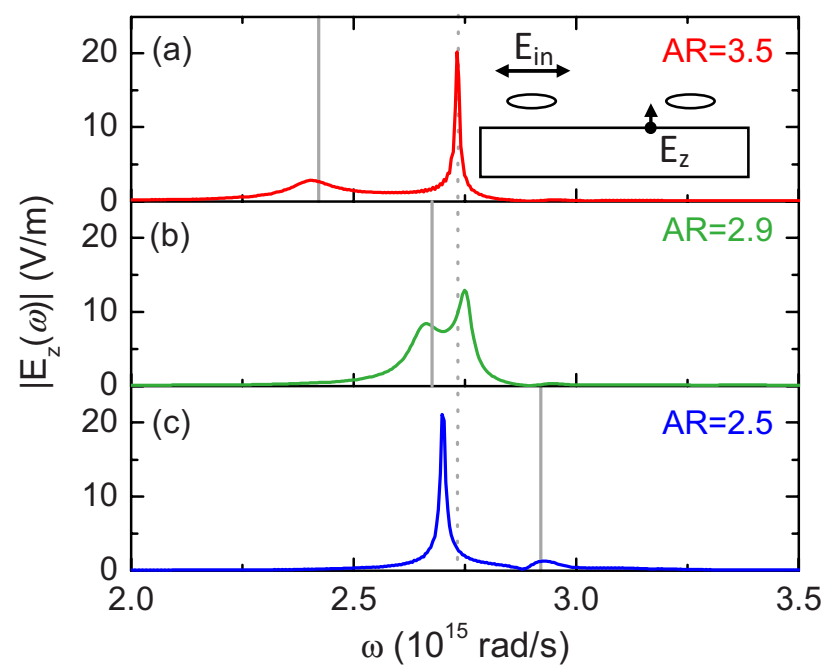

FIG. 1. (Color online) Electric field magnitude $\left|E_{z}(\omega)\right|$ at the indicated probe location, for (a) $\mathrm{AR}=3.5$, (b) $\mathrm{AR}=2.9$, and (c) $\mathrm{AR}=2.5$, for a NP volume of $9.8 \times 10^{3} \mathrm{~nm}^{3}$. The solid vertical lines indicate the isolated NP resonance, and the vertical dashed line marks the grating resonance. 


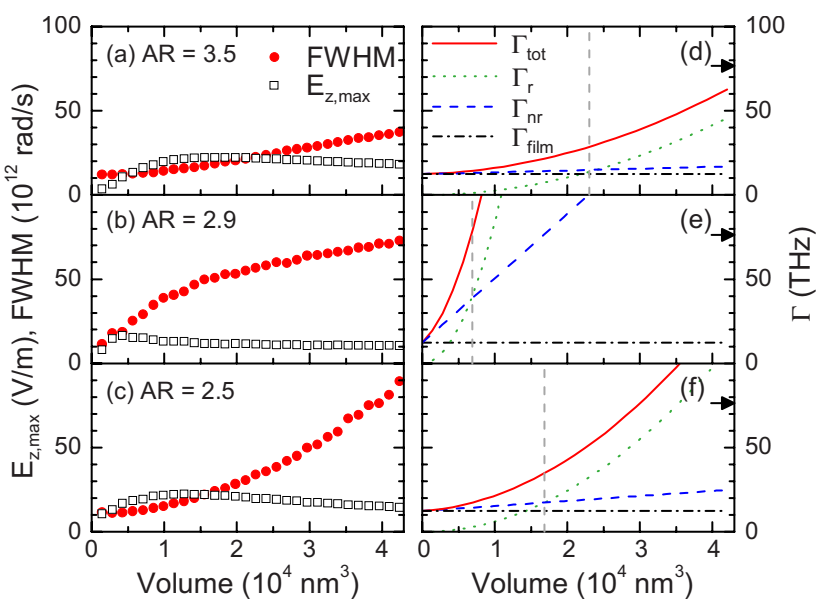

FIG. 2. (Color online) Peak electric field amplitudes of the grating resonance (open squares) and the FWHM of the grating resonance (filled circles) as a function of NP volume for NP ARs (a) 3.5, (b) 2.9, and (c) 2.5. Calculated radiative and nonradiative damping contributions to the total SP damping for (d) $\mathrm{AR}=4.05$, (e) $\mathrm{AR}=3.38$, and (f) $\mathrm{AR}=2.97$. The dashed vertical lines indicate the condition $\Gamma_{n r}=\Gamma_{r}$.

$2.73 \times 10^{15} \mathrm{rad} / \mathrm{s}$ ), and one related to SPs excited at frequencies close to the dipolar plasmon resonance of the NPs (broad resonance at $2.32 \times 10^{15} \mathrm{rad} / \mathrm{s}$ ). These features will be referred to, respectively, as the grating resonance and the NP resonance. Figures 1(b) and 1(c) show the corresponding results obtained for NPs with the same volume, but with an AR of 2.9 and 2.5, respectively. Since the particle resonance frequency is shape dependent, ${ }^{14}$ the shapes studied cover representative cases in which the particle resonance frequency is well below $(\mathrm{AR}=3.5)$, near $(\mathrm{AR}=2.9)$, and well above $(\mathrm{AR}=2.5)$ the grating resonance frequency.

To study the influence of the NP volume on SP excitation, the calculations shown in Figs. 1(a)-1(c) were repeated for NP volumes in the range of $1.4 \times 10^{3}-4.2 \times 10^{4} \mathrm{~nm}^{3}$. The SP electric field amplitude at the grating resonance $E_{z, \max }$ and the full width at half maximum (FWHM) of the grating resonance peak were obtained for all three NP shapes by performing a two-peak Lorentz fit for the data obtained from the simulations. For the case where the particle and grating resonance frequencies are almost equal $(\mathrm{AR}=2.9)$, the narrow peak closest to the predicted grating resonance frequency was analyzed. Figures 2(a)-2(c) shows $E_{z, \max }$ (open squares) and the FWHM (filled circles) for the grating resonance as a function of $V_{\mathrm{NP}}$ for the three NP ARs. For NPs with $\mathrm{AR}=3.5$ in the low-volume limit [Fig. 2(a)], the FWHM of the grating resonance is approximately constant and $E_{z, \max }$ increases approximately linearly with NP volume. At high volume $\left(V_{\mathrm{NP}}>\sim 1.5 \times 10^{4} \mathrm{~nm}^{3}\right) E_{z, \max }$ decreases slowly as $V_{\mathrm{NP}}$ is increased, while the FWHM increases significantly. At the transition between these two regimes, a maximum SP amplitude of $22 \mathrm{~V} / \mathrm{m}$ is obtained. Similar trends are observed for ARs of 2.9 and 2.5 [Figs. 2(b) and $2(\mathrm{c})]$ with $E_{z, \max }$ peaking at $V_{\mathrm{NP}} \approx 0.4 \times 10^{4} \mathrm{~nm}^{3}$ and 1.4 $\times 10^{4} \mathrm{~nm}^{3}$, respectively. In all cases the onset of an increase in the FWHM coincides with the occurrence of a maximum in $E_{z \text {,max }}$. In addition, as $V_{\mathrm{NP}}$ approaches zero, the FWHM of all the grating resonances converge to $\sim 1.17 \times 10^{13} \mathrm{rad} / \mathrm{s}$. Note that the maximum $E_{z, \max }$ reached for the three cases is approximately $20.3 \pm 3.3 \mathrm{~V} / \mathrm{m}$. Perhaps surprisingly, these results suggest that the frequency of the NP resonance is not a critical parameter in the optimization of SP excitation in these large-area NP enhanced grating couplers.

While the NPs are necessary to convert the incident farfield radiation into near-fields that can excite SPs, they also introduce significant radiative and nonradiative damping of the propagating SPs. In the system under consideration, SP damping occurs due to (a) intrinsic resistive losses in the metal substrate, (b) nonradiative damping due to finite fieldoverlap of the SP modes on the film and the SP modes of the NPs resulting in NP-induced resistive losses, and (c) radiative damping via dipole radiation generated by the NPs. The latter occurs when the particles are placed approximately one plasmon wavelength apart, leading to constructive interference of the NP radiation along the surface normal.

The different radiative and nonradiative damping contributions can be calculated analytically. The intrinsic SP damping rate can be found from known expressions for the SP group velocity and propagation length. ${ }^{.}$For a $\mathrm{Ag} / \mathrm{SiO}_{2}$ interface this leads to a predicted field damping constant $\Gamma_{\text {film }}=1.24 \times 10^{13} \mathrm{~s}^{-1}$ at the center frequency used. The NPinduced contribution to the total damping can be estimated by considering analytically calculated optical cross-sections for the dipolar NP plasmon resonance. For an isolated NP illuminated along one of its principal axes by a plane wave with electric field amplitude $E$, the total scattered (radiated) power is $P_{r, \mathrm{NP}}=\sigma_{r} I=1 / 2 n c \varepsilon_{0} E^{2} \sigma_{r}$, where $\sigma_{r}$ is the radiative dipolar plasmon scattering cross-section for the incident electric field direction, $I$ is the incident irradiance associated with the incident electric field $E, n$ is the refractive index of the host material, $\varepsilon_{0}$ is the permittivity of vacuum, and $c$ is the speed of light. In order to determine an approximate value for the NP-induced radiative damping we consider the local electric field $E_{\mathrm{loc}}$ at the location of the NP caused by the presence of SPs on the metal film, assuming that the calculated $\sigma_{r}$ of the NP remains approximately valid in the presence of the metal substrate. Although this assumption excludes any influence of image charges in the substrate, it will be shown to lead to reasonable estimates for the FWHM of the grating resonance. For a given lateral electric field amplitude $E_{0}$ of the SP at the metal surface, the local lateral electric field $E_{\mathrm{loc}}$ at the NP height $z_{\mathrm{NP}}$ is $E_{\mathrm{loc}}=E_{0} e^{-k_{z} z_{\mathrm{NP}}}$ with $k_{z}$ the known normal component of the SP wavevector. Given this assumption, the total damping of the SP energy per unit cell, $U_{\mathrm{SP}}$, due to reradiation by a single $\mathrm{NP}$ is approximately given by $\left(d U_{\mathrm{SP}} / d t\right)_{\mathrm{rad}}=P_{r, \mathrm{NP}}=-1 / 2 n c \varepsilon_{0} E_{0}^{2} e^{-2 k_{z} z \mathrm{NP}} \sigma_{r}$. To convert this radiated power to a SP damping rate we compare this value to the $U_{\mathrm{SP}}(t)$ in a single unit cell of volume $V$ averaged over one optical cycle, given by $U_{\mathrm{SP}}(t)$ $=\int_{V} 1 / 2 \varepsilon_{0} \varepsilon(\vec{r}) E_{\mathrm{SP}}^{2}(\vec{r}, t) d V$. Here $\varepsilon(\vec{r})$ is the known positiondependent isotropic permittivity, and $E_{\mathrm{SP}}(\vec{r}, t)$ is the positiondependent electric field amplitude associated with a standing SP wave with an angular frequency $\omega$ equal to the center wavelength and a lateral wavevector $k_{x}=2 \pi / L_{x}$. In this analysis the decay rate of the SP amplitude is assumed to be slow compared to an optical cycle. The radiative damping rate is related to the energy loss through $\left(d U_{\mathrm{SP}} / d t\right)_{\mathrm{rad}}$ $=-2 \Gamma_{r, \mathrm{NP}} U_{\mathrm{SP}}(t)$, where the factor 2 reflects the fact that the damping rates in this study represent electric field decay rates. With these expressions for the total energy and the scattered power per unit cell, we can now calculate the effective radiative damping rate according to $\Gamma_{r, \mathrm{NP}}$ $=P_{r, \mathrm{NP}} / 2 U_{\mathrm{SP}}$. An analogous analysis is done to obtain a similar expression for the NP-induced absorption, based on the 
nonradiative scattering cross-section $\sigma_{n r}$. The cross-sections $\sigma_{r}$ and $\sigma_{n r}$ are calculated based on the Rayleigh-Gans theory $^{14}$ as $\sigma_{r}=k^{4}|\alpha|^{2} /(6 \pi)$ and $\sigma_{n r}=k \cdot \operatorname{Im}[\alpha]$, where $k$ $=2 \pi n / \lambda_{0}$ at the center wavelength $\lambda_{0}=676 \mathrm{~nm}$, and $\alpha$ is the complex polarizability of the NP, calculated using the dielectric functions for $\mathrm{SiO}_{2}$ and $\mathrm{Ag}$ that were previously used in the simulations. Note that this analysis leads to entirely analytical expressions for the estimated NP-induced radiative and nonradiative damping.

Using the analysis described above, NP-induced damping contributions were evaluated for NPs with ARs 4.05, 3.38 , and 2.97, corresponding to theoretical resonance frequencies close to the numerically obtained results in Figs. 2(a)-2(c). Relatively high ARs were needed to match the numerically obtained resonance frequencies, since the Rayleigh-Gans theory does not take into account finite NP size effects and NP-substrate interactions. Figures 2(d)-2(f) shows the calculated damping contributions for the three ARs considered. The total nonradiative damping [blue dashed lines in Figs. 2(d)-2(f)] is given by $\Gamma_{n r}=\Gamma_{n r, \mathrm{NP}}$ $+\Gamma_{\text {film. }}$. Figure 2(d) shows the calculated damping terms for $\mathrm{AR}=4.05$, in which case the NP resonance frequency is lower than the grating resonance frequency. In the limit $V_{\mathrm{NP}} \rightarrow 0$, we see that the total nonradiative damping is equal to $\Gamma_{\text {film. }}$. As $V_{\mathrm{NP}}$ increases, $\Gamma_{n r}$ is seen to increase linearly, while $\Gamma_{r}$ increases quadratically. For $V_{\mathrm{NP}}<2.3 \times 10^{4} \mathrm{~nm}^{3}$, nonradiative damping is found to be the dominant loss contribution, whereas for larger values of $V_{\mathrm{NP}}$ radiative damping quickly becomes dominant. The volume at which radiative damping becomes the dominant loss contribution is indicated by the dashed vertical lines in Figs. 2(d)-2(f). The dependence of the FWHM based on the estimated damping contributions mimics the observations made in the numerical results shown in Fig. 2(a), suggesting that NP-induced damping is the cause of the increased FWHM of the grating resonance in Fig. 2(a) at large $V_{\mathrm{NP}}$. Since the FWHM (units $\mathrm{rad} / \mathrm{s}$ ) of a narrow Lorentzian resonance is equal to the numerical value of $\Gamma\left(\mathrm{s}^{-1}\right)$, we may directly compare the calculated $\Gamma$ values in Figs. 2(d)-2(f) with the FWHM values in Figs. 2(a)-2(c). This comparison reveals that the approximate analytical calculations predict the correct order of magnitude for the FWHM, without the use of any free parameters. It is important to note that the analytical calculations described here can be used to evaluate the damping contributions in similar NP arrays of different materials and for different particle shapes by simply replacing the corresponding parameters (dielectric function, semiaxes $a, b$, and $c$ ). Additionally, particle-induced damping for a different grating period can be evaluated by repeating the calculations at the resonance wavelength corresponding to the new grating spacing, and analytically calculating the particle crosssections at this wavelength.

Similar trends are observed for NP ARs of 3.38 and 2.97. For these cases the volume at which the $\Gamma_{r}$ contribution becomes dominant can be seen to be $0.7 \times 10^{4}$ and 1.7 $\times 10^{4} \mathrm{~nm}^{3}$, respectively. These findings can be understood by realizing that the damping of the grating resonance depends on the NP-induced damping at the grating resonance frequency. When the NP resonance lies close to the grating resonance, the NP has a large radiative and nonradiative cross-section (i.e., appears "optically large"), leading to strong NP-induced damping at relatively low volume. Note that the presented model also correctly predicts that the total damping will always converge to $\Gamma_{\text {film }}$ in the limit of zero particle volume, as observed in the numerical simulations.

While the model calculations accurately predict the trends observed in Figs. 2(a)-2(c), the simulated volumedependent FWHM in Fig. 2(b) differs significantly from the corresponding calculated damping in Fig. 2(e) for NPs with a resonance close to the grating resonance frequency. At low volume the simulated FWHM follows a similar increase as the estimated FWHM in Fig. 2(e), but rapidly diverges from the simulated FWHM above a volume of $0.7 \times 10^{4} \mathrm{~nm}^{3}$. Above this volume, the calculated total NP cross-section exceeds the size of the unit cell, as marked by the arrow on the right axis. We therefore attribute the deviation from the calculated damping in Fig. 2(e) to the saturation of the NPinduced damping.

The use of isolated NPs is expected to be beneficial in cases where maximum field enhancement or maximum control over resonance frequencies is needed. For optimum excitation of unidirectional propagating SPs the use of suspended metal lines along the $y$-direction may present added advantages. In such systems a similar optimization of line volume and cross-sectional shape will be needed in order to balance line-induced SP excitation and damping.

In conclusion, we have demonstrated that the appearance of a maximum SP amplitude in NP-enhanced grating couplers is due to the combined effect of an increased excitation of SPs as the particle volume increases, counteracted by an increase in the particle-induced damping at increasing volume. Our results indicate that control over the NP resonance frequency does not strongly affect the SP excitation efficiency in large NP-enhanced grating couplers if the NP volume can be freely modified, but does affect the NP volume at which maximum SP excitation occurs.

This work was supported in part by the National Science Foundation (CAREER Award No. ECCS-0644228) and by the U. S. Army Research Office (Grant No. 50372-CHMUR).

${ }^{1}$ H. Raether, Surface Plasmons on Smooth and Rough Surfaces and on Gratings, Springer Tracts in Modern Physics (Springer, Berlin, 1988), Vol. 111.

${ }^{2}$ B. Steinberger, A. Hohenau, H. Ditlbacher, A. L. Stepanov, A. Drezet, F. R. Aussenegg, A. Leitner, and J. R. Krenn, Appl. Phys. Lett. 88, 094104 (2006)

${ }^{3}$ D. K. Gramotnev and D. F. P. Pile, Appl. Phys. Lett. 85, 6323 (2004).

${ }^{4}$ D. F. P. Pile and D. K. Gramotnev, Appl. Phys. Lett. 86, 161101 (2005).

${ }^{5}$ K. Leosson, T. Nikolajsen, A. Boltasseva, and S. I. Bozhevolnyi, Opt. Express 14, 314 (2006)

${ }^{6}$ J. A. Dionne, L. A. Sweatlock, H. A. Atwater, and A. Polman, Phys. Rev. B 73, 035407 (2006)

${ }^{7}$ G. Webb-Wood, A. Ghoshal, and P. G. Kik, Appl. Phys. Lett. 89, 193110 (2006).

${ }^{8}$ A. Ghoshal, G. Webb-Wood, C. Mazuir, and P. G. Kik, Proc. SPIE 5927, 592714 (2005).

${ }^{9}$ H. Ditlbacher, J. R. Krenn, A. Hohenau, A. Leitner, and F. R. Aussenegg, Appl. Phys. Lett. 83, 3665 (2003).

${ }^{10}$ N. Papanikolaou, Phys. Rev. B 75, 235426 (2007).

${ }^{11}$ A. Ghoshal and P. G. Kik, J. Appl. Phys. 103, 113111 (2008).

${ }^{12}$ A. Ghoshal, I. Divliansky, and P. G. Kik, Appl. Phys. Lett. 94, 171108 (2009).

${ }^{13}$ Computer Simulation Technology, Darmstadt, Germany (2008).

${ }^{14}$ C. F. Bohren and D. R. Huffman, Absorption and Scattering of Light by Small Particles (Wiley, New York, 1998). 